\title{
Monseigneur Courchesne, éducateur
}

\section{Saint-François-de-Borgia et Thérèse Bernier}

Volume 34, 1967

URI : https://id.erudit.org/iderudit/1007301ar

DOI : https://doi.org/10.7202/1007301ar

Aller au sommaire du numéro

Éditeur(s)

Les Éditions Historia Ecclesiæ Catholicæ Canadensis Inc.

ISSN

0318-6172 (imprimé)

1927-7067 (numérique)

Découvrir la revue

Citer cet article

Saint-François-de-Borgia \& Bernier, T. (1967). Monseigneur Courchesne, éducateur. Sessions d'étude - Société canadienne d'histoire de l'Église catholique, 34, 9-17. https://doi.org/10.7202/1007301ar

Tous droits réservés @ Les Éditions Historia Ecclesiæ Catholicæ Canadensis Inc., 1968
Ce document est protégé par la loi sur le droit d'auteur. L'utilisation des services d'Érudit (y compris la reproduction) est assujettie à sa politique d'utilisation que vous pouvez consulter en ligne.

https://apropos.erudit.org/fr/usagers/politique-dutilisation/ 


\section{Monseigneur Courchesne, éducateur}

L'éducation est ce travail par lequel des hommes, mûris et dans le plein développement de leurs facultés, aident l'enfant à se développer à son tour et le conduisent à sa destinée pour cette vie et pour l'autre ${ }^{1}$.

Conduire les autres à l'épanouissement de leur vie terrestre et à la béatitude finale, telle apparaît la mission de Georges Courchesne; il s'acquitte de cette mission en réponse au mandat divin. "Allez, enseignez. En l'accomplissant, il trouve sa propre joie, car on dirait qu'il est fait pour cela, enseigner.

Prêtre humaniste, tout ce qui est humain l'intéresse : l'homme dans les manifestations de son esprit, les lettres, les sciences, les arts; l'homme dans les plus modestes œuvres de ses mains, l'agriculture, le commerce, l'industrie; l'homme spirituel dans sa démarche vers les biens éternels. Son activité d'éducateur s'est exercée à travers tous les domaines de l'humain.

Sans minimiser l'importance des remarquables initiatives de Georges Courchesne, dans les autres domaines, nous nous attacherons spécialement à l'un des aspects de sa carrière. Après avoir brièvement présenté l'homme, nous évoquerons les traits de l'éducateur, de l'écrivain et du professeur.

Parler de l'éducateur c'est circonscrire presque toute la vie du prêtre, de l'évêque, de l'archevêque dont la mission essentielle a été d'enseigner soit par la parole, soit par la plume.

Né le 13 septembre 1880, d'une famille de cultivateurs de Pierreville, Georges Courchesne grandit durant une période tumultueuse de l'histoire de l'Eglise canadienne, celle des luttes ultramontaines. L'enfant fréquente d'abord l'école primaire, dans le rang du Chenal Tardif, puis l'Académie de son village ${ }^{2}$. La vivacité de son intelligence, le bel équilibre de ses facultés étonnent ses maîtres qui se demandent : Que sera cet enfant ?

En 1892, au Séminaire de Nicolet, il devient vite au milieu de ses condisciples Bernier, Lahaie et autres, l'étoile de la classe ${ }^{3}$. Il sera certainement un grand homme, dit-on dans son entourage. En 1901, Georges Courchesne se destine au sacerdoce; il poursuit ses études théologiques à Nicolet, s'appliquant à la fois à l'étude et à l'enseignement comme c'était la coutume à l'époque.

1 Georges Courchesne, Nos Humanités, Nicolet, 1927, 720 pages, Intr.: 4.

2 Arthur Bergeron, ptre curé, Pierreville 1853-1953, un siècle de vie paroissiale et l'aurore du suivant, 1960, $246 \mathrm{p}$.

3 Francis GoYkR, Mgr Courchesne, dans Revue eucharistique du clergé, Montréal, 21 novembre 1950; 624-632. 
Il est ordonné le 10 juillet 1904, dans la chapelle du vieux Séminaire de Nicolet, par Son Excellence Mgr Bruneault, second évêque du diocèse. Et c'est le début d'une fructueuse carrière de professeur. Durant quatre ans, le jeune prêtre acquiert auprès des rhétoriciens du Petit Séminaire de Nicolet une expérience pédagogique qui le prépare à des études subséquentes.

Conscients de ses remarquables dons pour l'enseignement, ses supérieurs lui donnent un congé d'études à Rome, en 1908. Il s'adonne à la théologie sans pour autant négliger les arts et la littérature. Ses conférences littéraires, par la suite, porteront la marque d'un goût esthétique affiné au contact de l'art italien. Ses disciples se rappellent avec enthousiasme l'enseignement donné dans une magistrale leçon sur l'éducation de la sensibilité par la culture esthétique ${ }^{4}$.

En 1909, docteur en théologie, l'abbé Courchesne quitte Rome pour continuer des études pédagogiques à l'Université de Fribourg complétant par les voyages et les contacts avec les gens de mentalités diverses la formation psycho-pédagogique qui le servira tant dans l'éducation de la jeunesse que dans la direction de son diocèse.

Auréolé d'un prestige nouveau, après un séjour en Terre Sainte, où il complète sa formation, Georges Courchesne revient à Nicolet en 1911. Cependant sa belle physionomie ne perd rien de sa noble simplicité. Grand, le front harmonieux, la bouche spirituelle, le menton volontaire, l'abbé Courchesne incarne aux yeux de ses élèves de rhétorique l'idée du Maître que l'on admire et que l'on suit. Façonnée aux excellentes méthodes, l'intelligence du professeur ne se borne pas à préparer des bacheliers satisfaits, mais elle donne le goût de la culture littéraire; par la constance, le travail et la réflexion, elle ouvre devant les jeunes talents les séduisantes avenues du savoir. Le jeune maître pratique ses propres théories :

Evitons une critique tâtillonne, plus propre à réprimer qu'à épanouir l'émotion. On ne produira jamais rien en éducation, si l'on est victime d'un sens critique exagéré. L'enfant a surtout besoin qu'on l'enseigne, non pas séchement comme un manuel, mais de façon vivante et sentie, indicative plus que dogmatique... Si j'osais, j'irais jusqu'à dire : même dans l'enseignement des sciences mathématiques, physiques, sachons découvrir aux élèves ce que le vrai offre de splendeur. Il $\mathrm{y}$ a dans les sciences une poésie qu'il appartient au maître d'évoquer : poésie du chercheur qui se consacre à la conquête de la vérité et poésie de cette connaissance même qu'ont goûtée les plus grands génies ${ }^{5}$.

Maître en pleine possession de son talent, l'abbé Courchesne ne voyage pas à l'aventure, indifférent à l'équipage; toute sa classe se sent prise à bord, lorsque au début du cours il promène son regard d'aigle sur l'assistance. Il ne travaille pas non plus dans l'abstrait, hors du temps et de l'espace :

4 Georges Courchesne, ptre, Explication du tableau de Raphaël, La Trans. figuration, conférence donnée à l'Ecole normale de Nicolet, août 1914, 13 p.

5 Georges Courchesne, ptre, Conférence pédagogique donnée à l'Ecole normale de Nicolet, août 1914, 10 p. : 7. AMMN. 
Il sait que ses élèves appartiennent à un pays, à une race, à un temps déterminé, qu'ils sont les héritiers d'une langue, d'une civilisation particulières, qu'ils auront à vivre dans des conditions que ne connaissent pas, par exemple, les Français d'Europe ${ }^{6}$.

Ces divers facteurs entrent en ligne de compte dans ses exposés. Emaillées tantôt de traits d'esprit, tantôt d'anecdotes pertinentes ou de pensées lapidaires, ses leçons atteignent à des profondeurs d'où elles ne s'évadent plus. Il faut que tous les élèves participent au voyage de la connaissance entraînés par un tel Maître. Chacun sait qu'il ne ferait pas bon se détacher du professeur au cours d'une explication; c'est lui qu'il fallait écouter, au risque, disait l'une de ses élèves, d'encourir le sort de la femme de Loth; aussi est-ce dans une atmosphère favorable qu'il applique ses principes pédagogiques : Science, méthode et dévouement ${ }^{7}$.

La science s'impose à l'abbé Courchesne, comme une loi de première importance en pédagogie; nous citons sa pensée à ce sujet :

Il suffit d'indiquer la mission du professeur pour faire voir combien il devrait posséder les connaissances qu'il faut communiquer... Car il est par état un intellectuel transmetteur. Intellectuellement, il semble avoir un rôle médiocre, puisque sa tâche n'est pas de créer sa science, mais de ressasser des vérités connues. Socialement, son rôle est capital, puisque sans lui aucune science ne se transmettrait. Il est l'anneau intellectuel qui relie les générations ${ }^{8}$.

Pour apprivoiser la science, l'abbé Courchesne se livre constamment à l'étude. Les collègues du Séminaire le trouvent toujours au milieu des livres, quand ils le cherchent, durant les vacances ${ }^{9}$. Mais la science ne lui suffit pas, il vise à la méthode ${ }^{10}$. Ouvrons au hasard n'importe laquelle de ses conférences, lisons l'un de ses discours, toujours nous sommes captivés par la méthode du maître. Il considère l'enseignement comme un art difficile, aussi la conférence universitaire, le cours de rhétorique sont-ils préparés avec un soin égal. Semble-t-il se jouer avec les procédés de la méthode, ce n'est pas l'effet du hasard, mais le résultat d'une rigoureuse sélection de matériaux, par respect, dit-il à une collaboratrice, pour l'intelligence des auditeurs. Selon l'abbé Courchesne, science et méthode ont une cause efficiente qui s'appelle le dévouement :

Pour acquérir cette science qu'il faut communiquer aux enfants; pour donner cette science d'une manière accessible aux intelligences diverses; pour bien faire la préparation de classe, pour voir à la correction des devoirs; pour s'initier à tous ces moyens et procédés si précieux et qui rendent l'enseignement agréable en même temps qu'efficace; il faut du travail, et qui dit travail, dit dévouement 11 .

Dévoué, tel apparaît encore l'abbé Courchesne à travers la correspondance de ses anciens élèves. On le consulte à tout propos et spéciale-

6 Omer HÉroux, Mgr Courchesne, dans Le Devoir, 3 février 1928.

Georges Courchesne, Nos Humanités, L : 21.

Ibid., 20.

Mgr Paul MaYrand, Nicolet.

10 Georges Courchesne, op. cit., I : 21.

11 Georges Courchesne, Le dévouement, conférence donnée à un cercle d'étude de normaliennes, Ecole normale, Nicolet, (s.d.) AMMN. 
ment sur le choix d'un état de vie. Un indiscipliné qui avait dû permuter d'un collège à l'autre se rend compte du chagrin qu'il a causé à sa mère; il conserve aussi un sentiment d'admiration à l'égard de son ancien professeur, il lui écrit et le supplie d'envoyer quelques mots de consolation à sa mère ${ }^{12}$.

Parfois c'est aux prises avec les difficultés financières qu'on appelle au secours. Un étudiant en droit écrit à son ancien professeur, le 8 février 1913 :

Vous me dites : S̀ jamais tu avais besoin d'argent fais-moi le plaisir

de m'en parler. Je me résous à contre cœur à vous faire ce plaisir ${ }^{13}$.

Dans la lettre suivante, l'étudiant accuse réception d'un chèque substantiel. En termes émouvants il remercie son bienfaiteur le sachant, dit-il, obligé de gagner sur ses loisirs, le bonheur de rendre service. Il espère ne pas toujours être étudiant, dit-il, et pouvoir, à son tour, manifester sa reconnaissance à son professeur de Nicolet. Le dévouement désintéressé peut seul former des gens capables de nobles sentiments.

D'origine terrienne, l'abbé Courchesne est l'un de ceux qui croient à la vocation agricole des Canadiens français. Aussi cherche-t-il parmi ses élèves à déceler les aptitudes et à orienter vers l'agronomie les fils de cultivateur afin qu'il y ait des gens instruits parmi la population agricole de son pays. L'exemple le plus frappant est celui de feu le Lieutenant-gouverneur Paul Comtois. Les lettres de ce dernier à son professeur indiquent l'ascendant exercé par le maître sur son disciple ${ }^{14}$.

Nous pourrions allonger la liste des protégés de ce dévoué professeur.

Educateur des jeunes, l'abbé Courchesne l'est aussi des adultes par la prédication. Un jour, au Séminaire de Nicolet, il prêche durant une heure, devant son Evêque, les prêtres et les élèves de l'institution. “Çà n'a pas été long » de s'exclamer Mgr Bruneault ${ }^{15}$. C'était rendre hommage à son talent. En effet, il sait toujours quoi dire et comment le dire. On le demande partout. Il ne refuse jamais. Tantôt il donne un sermon de circonstance dans une église; tantôt il débite une conférence sur le romantisme dans un cercle littéraire; toujours oublieux de ses propres fatigues, il répond aux différents appels qui lui sont lancés. Les cadeaux qu'on lui offre alors constituent sa caisse de bienfaisance. Elle est toujours vide. Le dévouement, chez lui, se double de magnificence; cela convient à sa grandeur d'âme.

En lui, grandeur et bonté s'allient à une perspicacité qui lui fait découvrir le point vulnérable des hommes. Comme à bien des gens il lui arrive d'en abuser. Plus d'un confrère, certain jour, a ressenti l'acuité d'une pointe appliquée au bon moment et qui désarme l'adversaire. Certains en ont souffert parfois. Les plus grands hommes ne sont pas dépourvus de ces petits travers. Le rythme de travail qu'il s'impose

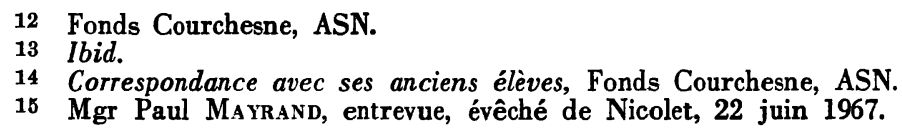


l'oblige à déposer les armes en 1917. S'éloignant pour un temps de son champ d'apostolat, il entre dans le ministère paroissial aux Etats-Unis. Homme d'études autant qu'homme d'action, l'abbé Courchesne profite de son séjour dans la république américaine pour scruter les problèmes des Franco-Américains. Il participe aux réunions d'action française. Il félicite les fondateurs de la Société historique franco-américaine, Edmond de Nevers, Albert Larue, le juge Dubuc et les autres. La société historique franco-américaine, dit-il, ne prétend pas être un instrument d'action populaire. Elle se recrute parmi une élite, parmi ceux qui suivent le mouvement des idées tout en étudiant le passé ${ }^{16}$.

Le séjour aux Etats-Unis exerce une salutaire influence sur la santé de l'abbé Courchesne, il revient bientôt à Nicolet pour œuvrer dans un nouveau champ d'action. Nommé Principal de l'Ecole normale de Nicolet, le $1^{\text {er }}$ juillet 1919 , le professeur se replonge dans les activités pour lesquelles on le dirait né. Continuer ses prédécesseurs; imprimer, autant que possible, un essor nouveau à l'institution; promouvoir la formation professionnelle des institutrices; voilà de quoi tenter un grand éducateur. Voyons-le au travail. Professeur méthodique et clair, il entraîne bientôt tous les professeurs de l'Ecole normale à la conquête du dépassement. Ses conférences aux Religieuses sont de véritables cours de formation pédagogique ${ }^{17}$. Il exige des professeurs de l'Ecole normale des travaux documentés sur des sujets d'histoire, d'art, de littérature, de philosophie ${ }^{18}$. Souvent invités à s'exécuter sous le regard sévère du Principal, les professeurs doivent apprendre à maitriser leurs matières.

Sous l'impulsion d'une volonté tenace, le niveau des études s'élève, la bibliothèque croît en importance, les cours de perfectionnement s'organisent, les cercles d'études fonctionnent, le Principal les considère comme le meilleur moyen de préparer l'élève-maître à la recherche des idées et comme une école de formation intellectuelle et morale. Tout son travail à l'Ecole normale revêt un souci constant de formation intégrale des institutrices et, par elles, celle des enfants des cours primaires.

Si nous analysons les résultats de l'activité intensive du chef, nous devons tirer des conclusions favorables : personnel compétent, discipline admirable, suivant l'adage de l'époque : tant vaut la discipline, tant vaut l'école; travail scolaire parfait, éducation soignée; bon nombre d'institurices remarquables.

Nous avons interrogé des normaliennes de cette époque. Toutes vantent la science et le savoir-faire de leur Principal; mais toutes, également, insistent sur l'austère rigueur du maître qui pousse les esprits jusqu'aux confins de la réflexion.

16 Georges Courchesne, Une soirée d'action française à Boston, dans L'Action française, $2^{\circ}$ année, $\mathrm{n}^{\circ} 11$, novembre 1918, p. 510-519: 515.

17 Georges Courchesne, Conférences littéraires, Ecole normale, Nicolet, 1920, AMMN.

18 Annuaire de l'Ecole normale de Nicolet, 1918-1928. 
L'abbé Courchesne souhaite de plus, que les institutrices soient des modèles de civisme, il n'hésite pas à leur rappeler les lois sévères du protocole. On raconte que l'abbé Courchesne étant devenu chanoine honoraire le $I^{\text {er }}$ décembre 1923 ; lorsque, pour la première fois, les élèves de l'Ecole normale le voient, revêtu de violet, la curiosité l'emportant sur la piété, elles ont l'audace de tourner la tête dans la cathédrale pour le regarder dans le cortège de ses pairs, leur geste reçut une sévère correction. Toutes celles que j'ai interrogées m'ont raconté l'incident. Les moindres détails sont utilisés par ce maître éducateur, quand il les considère favorables au développement des personnalités.

Une religieuse, collaboratrice de Monsieur le Principal Courchesne dit : Ma vie n'aurait pas suivi la même direction, si je n'avais pas eu l'avantage d'entrer en contact avec un tel éducateur.

Madame Françoise Gaudet-Smet, normalienne de ce temps-là, écrit au lendemain du décès de Mgr Courchesne :

A qui irons-nous maintenant ? Dans nos souvenirs vivifiés de sa sève, pour garder nos racines de foi, d'espérance et d'amour, si le sol et le sous-sol peuvent tenir le coup 19 .

Elle non plus n'a pas oublié le docte pédagogue de ses années de formation.

La réputation du Chanoine Courchesne traverse les murs de Nicolet. L'Ecole normale supérieure de l'Université Laval de Québec lui confie, en 1925, une chaire d'enseignement. Comme au Séminaire et à l'Ecole normale de Nicolet, le maître impressionne par ses qualités d'éducateur. Professeurs et étudiants réclament la publication d'un volume qui sera la somme pédagogique de cet éducateur.

Professeur remarquable, Mgr Courchesne est de plus éducateur par la plume. Son œuvre écrite est à la fois pédagogique, littéraire et pastorale. Euvre pédagogique, ce livre austère et scientifique est intitulé Nos Humanités. Ne fallait-il pas une profonde connaissance des théories pédagogiques de tous les temps pour fournir une synthèse aussi pleine? Ne fallait-il pas de plus l'acuité du regard de l'observateur pour parler des théories et des méthodes avec autant de réalisme? En 1927, le livre fait époque. Le monde intellectuel lui ménage un accueil flatteur. Les revues l'annoncent comme l'œuvre du jour et de l'époque. A l'âge de la science et de la technique, Nos Humanités aurait-il épuisé son message ? Nous ne croyons pas. Plus on avance dans la spécialisation, plus il faut recourir aux sources humanisantes comme celle de Nos Humanités.

A l'œuvre pédagogique publiée, il convient d'ajouter l'œuvre pédagogique restée dans les Archives et révélatrice des activités d'un pédagogue de chez nous. En effet, le Chanoine Courchesne a laissé des conférences sur les qualités du professeur, sur l'éducation esthétique,

19 Françoise Gaudet-Smet, Coup d'ail et de cour sur Mgr Courchesne, dans $L e$ Canado-Américain, $\mathrm{n}^{\circ} 139$, novembre-décembre 1950, Manchester, N.-H., p. 3-4. 
sur l'explication littéraire, sur l'histoire de l'art et sur les commentaires des œuvres de Maîtres ${ }^{20}$. A ses éduqués, il a parlé de tout.

L'œuvre de Georges Courchesne déborde le domaine de la pédagogie. Il collabore à l'Action française, à l'OEuvre des tracts. Cette dernière lui doit plusieurs bonnes brochures dans lesquelles les conférences se déroulent au rythme large d'une pensée ordonnée selon une harmonieuse logique et dans une langue où la clarté et l'éclat se disputent la première place. Ses œuvres trahissent le professeur possédé par le sujet et dont le charme et la densité de pensée captivent et retiennent les lecteurs.

Nos légitimes aspirations, paru en 1919, indique les relations francoaméricaines et le nationalisme du Chanoine Courchesne. L'auteur y définit l'idée de patrie, les devoirs impliqués dans cette idée, la pensée française et l'âme américaine, le catholicisme et les besoins de la démocratie, les catholiques d'origine française et le sens de la fraternité humaine. Ayant pénétré les replis de l'âme franco-américaine, il dit certaines vérités cruelles. Lisons plutôt :

L'obstacle principal à la survivance des qualités ethniques et de la langue française chez les Franco-Américains ne viendra pas surtout de ceux qui ne parlent que l'anglais, ou des pouvoirs publics au moins d'une façon générale. Il sera plutôt dressé par l'apathie de ceux qui volontiers s'en tiendraient à la loi du moindre effort. L'obstacle viendra encore de ceux qui considèrent comme une régression la fidélité du souvenir, et qui pensent que le progrès consiste uniquement à préparer les succès sans s'occuper du passé, de ses traditions et des lumières qu'il projette sur l'avenir 21.

Patriote et apôtre, il écrit pour les jeunes de son pays. Paraissait en 1925, une brochure intitulée l'Association catholique de la jeunessecanadienne-française; cette association, dit-il, doit être une école de perfection au service de la vie catholique, de la vie sociale et de la vie nationale ${ }^{22}$.

Reconnaissant les mérites de ce prêtre remarquable, l'Eglise l'élève à l'épiscopat. Sacré quatrième évêque de Rimouski, par Son Eminence le Cardinal Rouleau, le 24 mai 1928, Mgr Courchesne continue d'enseigner. Les brochures pastorales portent le message évangélique par delà les limites de son diocèse. En 1934, l'CEuvre des Tracts publie Quelques réflexions sur l'apostolat laïque, œuvre d'avant-garde, invitation au laïcat à prendre sa place dans l'Eglise. En 1942, paraît Qu'est-ce qu'un catholique pratiquant? L'évêque énumère, dans cet opuscule, les signes auxquels l'Eglise reconnaît ses vrais disciples. Mais l'œuvre littéraire capitale de l'évêque, ce sont les Circulaires et les Mandements adressés à son peuple et à son clergé. Ces longues lettres indiquent les sommets où se complaît la pensée de l'auteur : Dieu, L'Eglise, le bien des hommes. Nouvelles réponses de l'apôtre au mandat divin : « Allez,

20 Georges Courchesne, Conférences pédagogiques données aux religieuses de l'Ecole normale de Nicolet, AMMN.

21 Georges Courchesne, Nos légitimes aspirations, tract $\mathrm{n}^{\circ}$ 6, de la Ligue du ralliement français en Amérique, Montréal, 1919, 24 p. : 23.

22 Georges Courchesne, L'Association catholique de la jeunesse canadiennefrançaise, Euvre des Tracts, $n^{\circ}$ 77, Montréal, 1925, 16 p. : 1. 
enseignez ..." ", ses épîtres nous le montrent à la fois docteur, apôtre et père.

Docteur, il faut qu'il proclame la vérité. Le 2 février 1943, il écrit :

Toute vérité est à nous, et certes ce n'est pas l'Eglise qui a peur de la vérité et des vérités que la science humaine a pu trouver. L'Eglise voit même dans l'étude des êtres que Dieu a faits, l'étude de l'univers, un noble emploi des facultés humaines. Et c'est pourquoi nous attachons tant de prix aux études de nos institutions scolaires et aux études que vous ferez de plus en plus dans vos familles pour maîtriser votre professiữin?

Apôtre, il parle tantôt en faveur des cercles d'Action catholique, tantôt en faveur de l'UCC, souvent pour l'Association des institutrices car dit-il :

Le sort de l'école dépend du sérieux de l'institutrice. Le cercle pédagogique lui garde le goût de l'étude et guide son apostolat auprès des jeunes 24 .

Il s'inquiète de l'éducation des enfants et de celle des adultes. - Je crois, dit-il, qu'on devra se persuader de plus en plus que ce sont nos adultes qu'il faut surtout organiser et mettre à l'étude ${ }^{25}$...

Son zèle, sa volonté de promouvoir l'éducation dans son diocèse ont fait de ce grand évêque un créateur d'institutions alimentant tous les secteurs de la vie diocésaine : agriculture, commerce, industrie. La classe des travailleurs trouve en lui un paternel défenseur : L'Eglise, dit-il, ne peut se désintéresser des conditions matérielles, temporelles où la vie surnaturelle doit s'épanouir. Educateur de son peuple, il tient compte de tous, mais surtout des plus nécessiteux. Ferme appui de l'UCC durant plus de vingt ans, à sa façon dynamique, il a invité les agriculteurs à se mêler de leurs affaires. L'aphorisme Mêlez-vous de vos affaires, mais mêlez-vous-en lancé pour stimuler à l'action a couru sur les lèvres. Créateur de paroisses rurales, il s'inquiète du problème agricole. L'éducation peut le résoudre, pense-t-il. Il écrit en 1933 :

$\mathrm{Je}$ vous supplie de prendre des mesures pour suivre en entier ces séances d'études. (II parle de la Semaine sociale). Le problème agricole y sera examiné au point de vue économique. Vous aurez dans la pensée de l'examiner tout le temps au regard de la pratique des quatre vertus cardinales, qui constituent véritablement le bien commun 26 .

Il revient dans un mandement sur cette question agricole qui doit être l'objet de l'éducation. Il s'adresse aux institutrices. Il les engage à choisir les travaux scolaires dans les textes inspirés par les réalités de la vie rurale. Aux parents, il conseille d'éviter toute plainte contre la difficulté de leur état; à l'Ecole d'agriculture de développer les convictions autant que le savoir ${ }^{27}$. Les agriculteurs voient dans leur chef spirituel, non seulement un défenseur de leurs intérêts éternels mais encore un auxiliaire de leur bien-être humain.

23 Circulaire au clergé, 2 février 1943, Rimouski, IV : 4.

24 Ibid., 15 août 1943 : 31.

25 Ibid., 33.

26 Lettre sur l'A.S.C., 5 juillet 1933.

27 Mandements et Circulaires, I, mai 1928 à janvier 1936: 38. 
Promu archevêque le 11 février 1946, Mgr Courchesne écrit encore et toujours pour enseigner son peuple.

Educateur apôtre, son ambition consiste à rejoindre jusqu'au dernier de ceux dont il a la charge. Educateur de grande classe, par l'élévation de sa pensée, il entraîne l'adhésion des esprits et détermine les volontés à l'action. Jusqu'à sa mort survenue en 1950, il demeure d'un merveilleux enthousiasme pour les causes qu'il défendait. Il croit en la grandeur de l'homme puis en celle du monde. Convaincu, comme Teilhard de Chardin, que tout enrichissement humain n'est que poussière s'il ne s'agrège à un centre d'amour immortel, Mgr Courchesne éducateur a déployé toutes les forces de persuasion de la parole et de la plume pour les causes auxquelles il avait consacré sa vie : la gloire de Dieu, le bonheur des hommes, ses frères, dans l'Eglise canadienne.

Sœur Saint-Françors-de-Borgia, Professeur au cours secondaire, Nicolet. Thérèse Bernier, S.A.S.V., B.A., M.A., 